
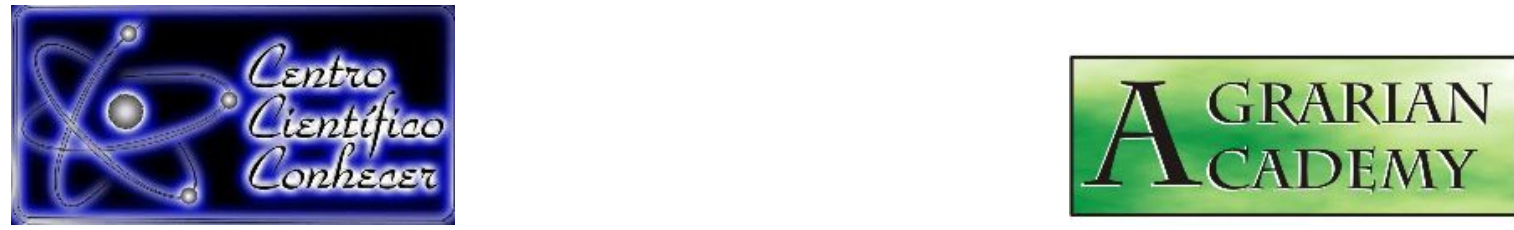

\title{
ESPACIALIZAÇÃO E CONCENTRAÇÃO DAS APLICAÇÕES DE CRÉDITO RURAL NO BRASIL ENTRE 2007 E 2017
}

\author{
Carina Chagas Madeira de Souza ; Marcos Antônio Souza dos Santos²; \\ Fabrício Khoury Rebello ${ }^{2}$
}
${ }^{1}$ Bolsista de Iniciação Científica (PIBIC/UFRA), graduanda do Curso de Agronomia da Universidade Federal Rural da Amazônia (UFRA), Belém/PA - Brasil.
${ }^{2}$ Doutor, Professor da Universidade Federal Rural da Amazônia (UFRA), Belém/PA - Brasil. (marcos.santos@ufra.edu.br)

Recebido em: 19/11/2018 - Aprovado em: 14/12/2018 - Publicado em: 25/12/2018 DOI: 10.18677/Agrarian_Academy_2018B9

\begin{abstract}
RESUMO
A política de crédito rural é um instrumento de significativa importância para o desenvolvimento rural, pois fornece recursos financeiros para a realização de operações que vão desde a implantação de atividades agropecuárias até a comercialização. Neste artigo, avaliou-se a distribuição espacial e a concentração do crédito rural no Brasil entre os anos de 2007 e 2017. Utilizou-se a base de dados do Banco Central do Brasil (BACEN, 2018) para calcular o Índice Normalizado de Crédito Rural (INCR) cujo resultado permite caracterizar a espacialização das atividades financiadas (agrícola ou pecuária) nas Unidades da Federação. Para verificar a concentração de crédito em ambos os segmentos produtivos calculou-se o Índice de Gini, considerando a quantidade e o valor das operações contratadas. Os resultados do INCR indicam que ocorreram algumas mudanças na distribuição espacial das aplicações de crédito em cada Unidade da Federação. As diferenças mais acentuadas foram percebidas nas regiões Norte e Nordeste, onde as aplicações de crédito foram maiores para a atividade pecuária, diferentemente da região Sudeste, onde os financiamentos foram mais voltados para atividades agrícolas. Concluiu-se que a concentração dos recursos de crédito rural no Brasil ainda é acentuada, pois $71,42 \%$ de todo o volume aplicado entre os anos de 2007 e 2017, foi alocado em apenas seis estados (PR, RS, SP, MG, GO e MT).
\end{abstract}

PALAVRAS-CHAVE: Desenvolvimento Rural, Financiamentos Agropecuários, Modernização da Agricultura, Política Agrícola.

\section{SPACIALIZATION AND CONCENTRATION OF RURAL CREDIT APPLICATIONS IN BRAZIL BETWEEN 2007 AND 2017}

\section{ABSTRACT}

He rural credit policy is a tool of great importance for the development in the field, since it provides the necessary financial capital for the accomplishment of operations 
that go from the implantation to the commercialization of products. In this paper, the spatial distribution and concentration of rural credit in Brazil in the period 2000-2017 is evaluated. For this purpose, the Central Bank of Brazil (BACEN) data base was used to calculate the Normalized Rural Credit Index (INCR), whose result allows to identify the spatialization of the activities financed (agricultural or livestock) in the study area. In order to verify the concentration of credit in both productive segments, the Gini Index was also calculated, taking into account the number of operation sand the value of contracting. The results of INCR made it possible to identify some changes in the specialization of credit applications in each unit of the federation. The most significant differences were observed in the North and Northeast regions, where credit applications were higher for livestock activities, differently from the Southeast region, where financing is more focused on agricultural activities. It is concluded that the concentration of rural credit resources in Brazil is still very marked, since $71.42 \%$ of the total volume applied between 2000-2017 was allocated in only 6 units of the federation (PR, RS, SP, MG, GO and MT).

KEYWORDS: Agricultural financing, Agricultural modernization, Agriculture policy, Rural development.

\section{INTRODUÇÃO}

A política de crédito rural é um dos principais instrumentos que incentivam o setor agropecuário, pois disponibiliza recursos financeiros para o custeio, investimento e comercialização de produtos agropecuários. Estes recursos contribuem para o aumento da produção e da produtividade, permitindo melhorias na qualidade de vida nas cidades e no meio rural (NASCIMENTO et al., 2011; SANTOS et al., 2012).

O crédito rural contribui para o processo de desenvolvimento rural que é fundamental para a segurança alimentar e qualidade de vida das populações, pois se configura como um dos elementos essenciais para a construção de uma agricultura eficiente, rentável e sustentável. Esta eficiência está relacionada com o nível de renda das famílias rurais, pois a partir do incremento financeiro dos produtores, ocorrerá a potencialização da escala de produção. Assim, os produtores podem buscar novos conhecimentos que contribuam para um desenvolvimento social e cultural (ANTÃO; CAMPANHOLO, 2011).

Em âmbito nacional, o crédito rural assumiu maior importância no desenvolvimento rural a partir da década de 1960, quando houve a criação do Sistema Nacional de Crédito Rural (SNCR). Estruturalmente, este sistema disponibiliza recursos para três finalidades básicas: investimento, custeio e comercialização, cujos objetivos específicos envolvem o estímulo à adoção de novas tecnologias e insumos modernos e a garantia da oferta equilibrada de produtos ao longo do tempo. Inicialmente, todos estes propósitos foram alcançados. Entretanto, no caso dos pequenos agricultores o fortalecimento econômico não ocorreu de forma significativa (RAMOS; MARTHA JUNIOR, 2010; ARAÚJO, 2011).

Sobre essa questão, trabalhos como o de Souza et al. (2015) indicam que este sistema tem sido fortemente criticado por questões distributivas, pois se tem observado grandes disparidades em relação ao valor de crédito recebido nas diferentes regiões e estados do País.

Souza e Barbé (2014) relatam que tais desigualdades são percebidas, sobretudo, na concentração regional dos recursos, que na maioria das vezes se limita a regiões onde os produtores possuem uma elevada capitalização. Essa dinâmica desigual de industrialização em diferentes regiões é citada por Santos AGRARIAN ACADEMY, Centro Científico Conhecer - Goiânia, v.5, n.10; p. 832018 
(2010), que justifica esse comportamento estabelecendo uma relação com a forma em que ocorre a concessão de crédito, onde se beneficiam em maior parte, locais com boa capacidade de resposta a expansão e diversificação requeridas principalmente pelas agroindústrias. Ou seja, o crédito distribuído de forma deliberada, sem critérios significativos e controle de uso, foi um dos principais motivos que desencadearam a concentração do capital financeiro em determinados grupos sociais, haja vista que em regiões onde a demanda dos centros urbanos, agroindústrias e de exportações são menos recorrentes, a modernização foi menor.

Para Belik (2015), os agricultores familiares são menos favorecidos com tais políticas, isto porque a produção em uma área relativamente menor pode ser um fator de desvantagem para a garantia de empréstimos, por exemplo. No sentido de contribuir com esta discussão, o presente artigo teve por objetivo identificar e quantificar a distribuição espacial e a concentração do crédito rural no Brasil no período de 2007 a 2017. A partir destes resultados, busca-se verificar em quais Unidades da Federação tem sido alocada a maior parcela das operações de financiamento para a agricultura e pecuária, além de aferir o nível de concentração nas operações e o valor de crédito repassado para cada estado.

\section{MATERIAL E MÉTODOS}

No desenvolvimento deste trabalho, as fontes básicas dos dados foram 0 Anuário Estatístico do Crédito Rural do Banco Central do Brasil e a Matriz de Dados de Crédito Rural (BACEN, 2018), referente ao período de 2007 a 2017. Foram obtidas informações relativas ao número e valor das operações de crédito agrícola, pecuário e total, contratados nas 27 Unidades da Federação. Para eliminar o efeito da inflação sobre o valor das operações, utilizou-se o Índice Geral de Preços Disponibilidade Interna (IGP-DI) da Getúlio Vargas (FGV, 2018), tendo como base a média do ano de 2017.

A classificação e espacialização foi realizada em função da principal atividade financiada por meio do cálculo do Índice Normalizado de Crédito Rural (INCR), especificado de acordo com a Fórmula 1, conforme descrita por Toledo Neto e Couto Junior (2011):

$$
I N C R_{i}=\frac{C A_{i}-C P_{i}}{C A_{i}+C P_{i}}
$$

$$
\text { Em que: }
$$

$I N C R_{i}=$ Índice normalizado das aplicações de crédito rural da i-ésima Unidade da Federação;

$C A_{i}=$ Valor das operações de crédito aplicado na atividade agrícola na i-ésima Unidade da Federação;

$C P_{i}=$ Valor das operações de crédito aplicado na atividade pecuária na i-ésima Unidade da Federação.

Os valores do INCR variam entre -1 e 1 . As Unidades da Federação foram classificadas em pecuaristas $(-1 \leq \mathrm{INCR}<-0,5)$; medianamente pecuaristas $(-0,5 \leq$ INCR $<0,0)$; medianamente agrícolas $(0,0 \leq \mathrm{INCR}<0,5)$ e agrícolas $(0,5 \leq \mathrm{INCR} \leq$ $1,0)$. Para aferir o nível de concentração das aplicações de crédito rural foi utilizado

AGRARIAN ACADEMY, Centro Científico Conhecer - Goiânia, v.5, n.10; p. 842018 
o índice de Gini (HOFFMAN, 1991), calculado por meio da Fórmula 2 a seguir:

$$
G=1-\sum_{k=1}^{n-1}\left(X_{k+1}-X_{k}\right) \times\left(Y_{k+1}+Y_{k}\right)
$$

Em que:

$\mathrm{G}=$ índice de Gini;

$\mathrm{X}=$ proporção acumulada do número de Unidades da Federação;

$Y=$ proporção acumulada de crédito rural liberado;

$\mathrm{n}=$ número total de Unidades da Federação.

A interpretação do índice de Gini é simples, pois quanto mais próximo de 1, maior o nível de concentração da distribuição do crédito rural.

\section{RESULTADOS E DISCUSSÃO}

A oferta de crédito pelo SNCR é segmentada em duas atividades básicas: agrícola e pecuária. Ao longo desta pesquisa, as análises dos índices de concentração avaliaram estes segmentos produtivos individualmente e em conjunto, sendo este último denominado "crédito total", pois representa o somatório dos valores referentes aos créditos destinados à agricultura e pecuária.

Os dados da Tabela 1 mostram a distribuição percentual do valor de crédito total entre as Unidades da Federação, considerando os anos 2007 a 2017. De acordo com os resultados, os estados com as maiores participações, foram: Rio Grande do Sul ( $R$ \$ 142 bilhões), São Paulo ( $R$ \$ 137 bilhões), Minas Gerais ( $R$ \$ 136 bilhões) e Paraná (R\$147 bilhões). Da mesma forma, os estados com as menores participações, foram: Amapá ( $\mathrm{R} \$ 229$ milhões), Roraima ( $\mathrm{R} \$ 665$ milhões), Amazonas ( $R \$ 1,6$ bilhões) e Distrito Federal (1,7 bilhões).

Em relação ao número de contratos realizados nesse período, as regiões Nordeste e Sul foram as que mais se destacaram. Porém, o fluxo destas operações seguiu padrões diferenciados. No caso do Nordeste, cerca de seis milhões de contratos foram destinados a pecuária. Diferentemente da região Sul, onde cinco milhões de operações atenderam a demanda agrícola (Figura 1).

TABELA 1. Valores percentuais da distribuição de crédito total (agrícola + pecuário) entre as Unidades da Federação nos anos 2007 a 2017.

\begin{tabular}{l|lllllllllll}
\hline UF & $\mathbf{2 0 0 7}$ & $\mathbf{2 0 0 8}$ & $\mathbf{2 0 0 9}$ & $\mathbf{2 0 1 0}$ & $\mathbf{2 0 1 1}$ & $\mathbf{2 0 1 2}$ & $\mathbf{2 0 1 3}$ & $\mathbf{2 0 1 4}$ & $\mathbf{2 0 1 5}$ & $\mathbf{2 0 1 6}$ & $\mathbf{2 0 1 7}$ \\
\hline AC & 0,13 & 0,09 & 0,11 & 0,16 & 0,11 & 0,19 & 0,38 & 0,40 & 0,38 & 0,44 & 0,41 \\
AP & 0,01 & 0,01 & 0,03 & 0,02 & 0,01 & 0,01 & 0,10 & 0,03 & 0,04 & 0,03 & 0,03 \\
AM & 0,22 & 0,18 & 0,20 & 0,14 & 0,10 & 0,19 & 0,26 & 0,17 & 0,16 & 0,09 & 0,15 \\
PA & 1,21 & 1,05 & 0,71 & 0,79 & 0,69 & 1,00 & 2,42 & 2,96 & 2,83 & 2,04 & 2,49 \\
RO & 0,61 & 0,47 & 0,64 & 0,80 & 0,69 & 0,90 & 2,28 & 3,29 & 3,50 & 3,53 & 3,88 \\
RR & 0,05 & 0,04 & 0,02 & 0,03 & 0,03 & 0,05 & 0,15 & 0,16 & 0,14 & 0,24 & 0,20 \\
TO & 1,07 & 1,15 & 1,17 & 1,21 & 1,30 & 1,68 & 2,86 & 3,20 & 3,73 & 2,64 & 2,99 \\
AL & 0,54 & 0,43 & 0,36 & 0,34 & 0,31 & 0,31 & 0,64 & 0,50 & 0,49 & 0,73 & 0,50 \\
BA & 3,19 & 3,26 & 3,27 & 3,41 & 3,73 & 3,89 & 4,43 & 4,19 & 4,24 & 4,00 & 3,42 \\
CE & 0,92 & 0,82 & 0,80 & 0,78 & 0,76 & 0,68 & 1,17 & 1,15 & 1,13 & 1,20 & 0,77 \\
MA & 1,32 & 1,33 & 1,28 & 1,28 & 1,19 & 1,14 & 2,23 & 2,40 & 2,20 & 2,31 & 2,18 \\
PB & 0,60 & 0,51 & 0,39 & 0,44 & 0,40 & 0,28 & 0,44 & 0,46 & 0,69 & 0,76 & 0,45 \\
PE & 1,02 & 0,76 & 0,66 & 0,64 & 0,59 & 0,60 & 0,96 & 1,04 & 1,02 & 1,09 & 0,68 \\
PI & 0,61 & 0,58 & 0,74 & 0,75 & 0,71 & 1,02 & 1,11 & 1,09 & 1,22 & 1,29 & 1,11
\end{tabular}

AGRARIAN ACADEMY, Centro Científico Conhecer - Goiânia, v.5, n.10; p. 85 


\begin{tabular}{c|ccccccccccc} 
RN & 0,46 & 0,34 & 0,24 & 0,23 & 0,26 & 0,24 & 0,42 & 0,43 & 0,48 & 0,51 & 0,32 \\
SE & 0,32 & 0,32 & 0,31 & 0,40 & 0,34 & 0,30 & 0,40 & 0,41 & 0,49 & 0,44 & 0,34 \\
ES & 2,09 & 1,83 & 2,09 & 2,09 & 2,02 & 1,92 & 2,15 & 2,38 & 1,65 & 1,72 & 1,60 \\
MG & 15,01 & 14,98 & 14,02 & 15,04 & 15,21 & 14,16 & 12,60 & 12,47 & 11,75 & 11,74 & 12,33 \\
RJ & 0,28 & 0,27 & 0,25 & 0,26 & 0,25 & 0,20 & 0,30 & 0,30 & 0,19 & 0,26 & 0,34 \\
SP & 18,48 & 16,37 & 18,47 & 15,62 & 14,77 & 13,04 & 9,68 & 8,71 & 7,89 & 9,28 & 8,24 \\
PR & 15,18 & 16,89 & 15,66 & 14,81 & 15,42 & 15,66 & 14,30 & 13,97 & 15,25 & 13,50 & 11,01 \\
RS & 14,38 & 16,12 & 15,98 & 16,69 & 15,40 & 14,69 & 12,40 & 11,91 & 11,42 & 11,51 & 11,64 \\
SC & 6,91 & 7,01 & 7,37 & 7,04 & 6,98 & 6,78 & 5,50 & 5,48 & 5,25 & 5,37 & 5,33 \\
DF & 0,21 & 0,21 & 0,21 & 0,27 & 0,19 & 0,16 & 0,16 & 0,10 & 0,09 & 0,06 & 0,07 \\
GO & 6,00 & 6,20 & 6,04 & 6,76 & 7,42 & 8,06 & 7,91 & 7,89 & 8,24 & 8,80 & 9,82 \\
MT & 5,13 & 4,88 & 5,06 & 5,82 & 6,75 & 7,70 & 9,43 & 9,96 & 10,37 & 11,14 & 13,30 \\
MS & 4,06 & 3,91 & 3,92 & 4,17 & 4,40 & 5,18 & 5,31 & 4,95 & 5,18 & 5,27 & 6,38 \\
\hline TOTAL & $\mathbf{1 0 0}$ & $\mathbf{1 0 0}$ & $\mathbf{1 0 0}$ & $\mathbf{1 0 0}$ & $\mathbf{1 0 0}$ & $\mathbf{1 0 0}$ & $\mathbf{1 0 0}$ & $\mathbf{1 0 0}$ & $\mathbf{1 0 0}$ & $\mathbf{1 0 0}$ & $\mathbf{1 0 0}$ \\
\hline
\end{tabular}

Fonte: dados da pesquisa.

FIGURA 1. Número total de contratos destinados à agricultura e pecuária por região, Acumulado 2007-2017.

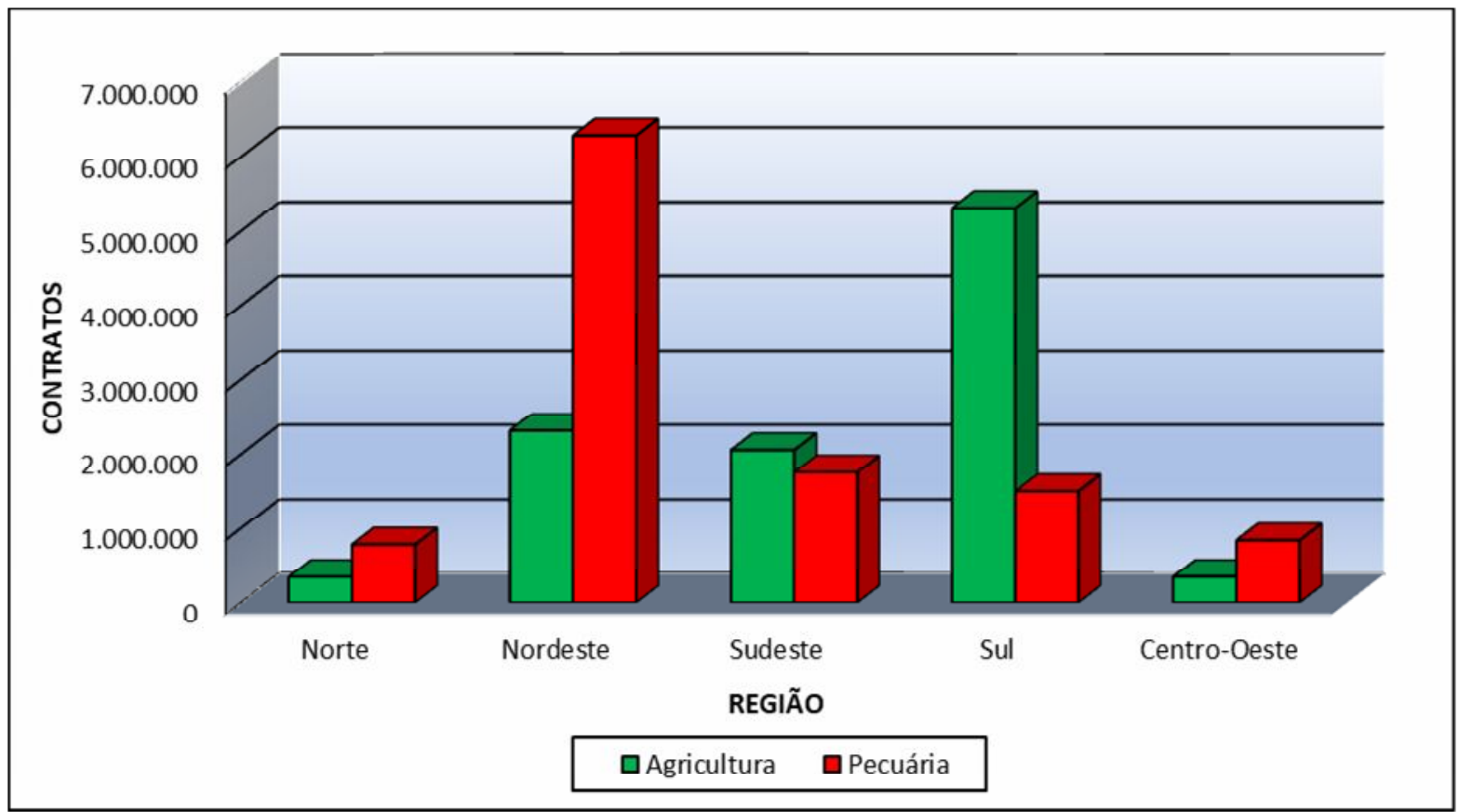

Fonte: dados da pesquisa.

A análise do INCR permitiu identificar a espacialização dos financiamentos destinados à agricultura e pecuária no Brasil e suas Unidades da Federação, para os anos de 2007 e 2017. Considerando os dados agregados para o País, percebeuse que, em 2007, o Brasil, quanto à alocação do crédito, assumia uma condição de mediamente agrícola, e, em 2017, passou para uma posição de mediamente pecuarista, indicando que o crédito passou a ser mais especializado na produção pecuária.

$\mathrm{Na}$ região Norte, no ano de 2007, quatro estados foram classificados como medianamente agrícolas ( $A C, A P, A M, R R$ ) e três estados classificados como medianamente pecuaristas (PA, RO, TO). No ano de 2017, ocorreram mudanças nesse cenário, quatro passaram a ser classificados como pecuaristas $(A C, R O, R R$, TO), dois se enquadraram em medianamente pecuaristas (AM, PA), sendo que 0 
Pará já ocupava essa posição no período anterior e apenas um estado agrícola (AP).

$\mathrm{Na}$ Região Nordeste, os resultados mostraram mudanças mais acentuadas. No ano de 2007, sete estados foram classificados como medianamente pecuaristas (AL, BA, CE, MA, PE, PI, SE) e dois como pecuaristas (PB, RN), em 2017, todos passaram a ser classificados como pecuaristas.

A região Sudeste, em 2007, apresentou um número elevado de financiamentos agrícolas. Isto porque de acordo com o INCR, três estados foram classificados como medianamente agrícolas (MG, RJ, SP) e um como agrícola (ES). Em 2017 a situação mudou, visto que a região passou a incorporar mais fortemente as políticas de crédito voltadas para a produção animal e por isso, em 2017, dois estados foram classificados como medianamente pecuaristas (MG e RJ), um medianamente agrícola (SP) e um agrícola (ES).

$\mathrm{Na}$ região Sul, todos os estados (PR, RS, SC) foram classificados como agrícolas em 2007. No entanto, em 2017 a região se diversificou, pois dois estados passaram para o nível de medianamente agrícolas (PR, RS) e um para medianamente pecuarista (SC). Por fim, o Centro-Oeste foi a única região que não apresentou diferença quanto à classificação do INCR, pois o Distrito Federal foi classificado como agrícola e três estados medianamente pecuaristas (GO, MT, MS), em ambos os períodos de análises. Todos estes resultados estão ilustrados na Figura 2.

FIGURA 2. Espacialização das Unidades da Federação do Brasil de acordo com o Índice Normalizado de Crédito Rural (INCR), 2007 e 2017.

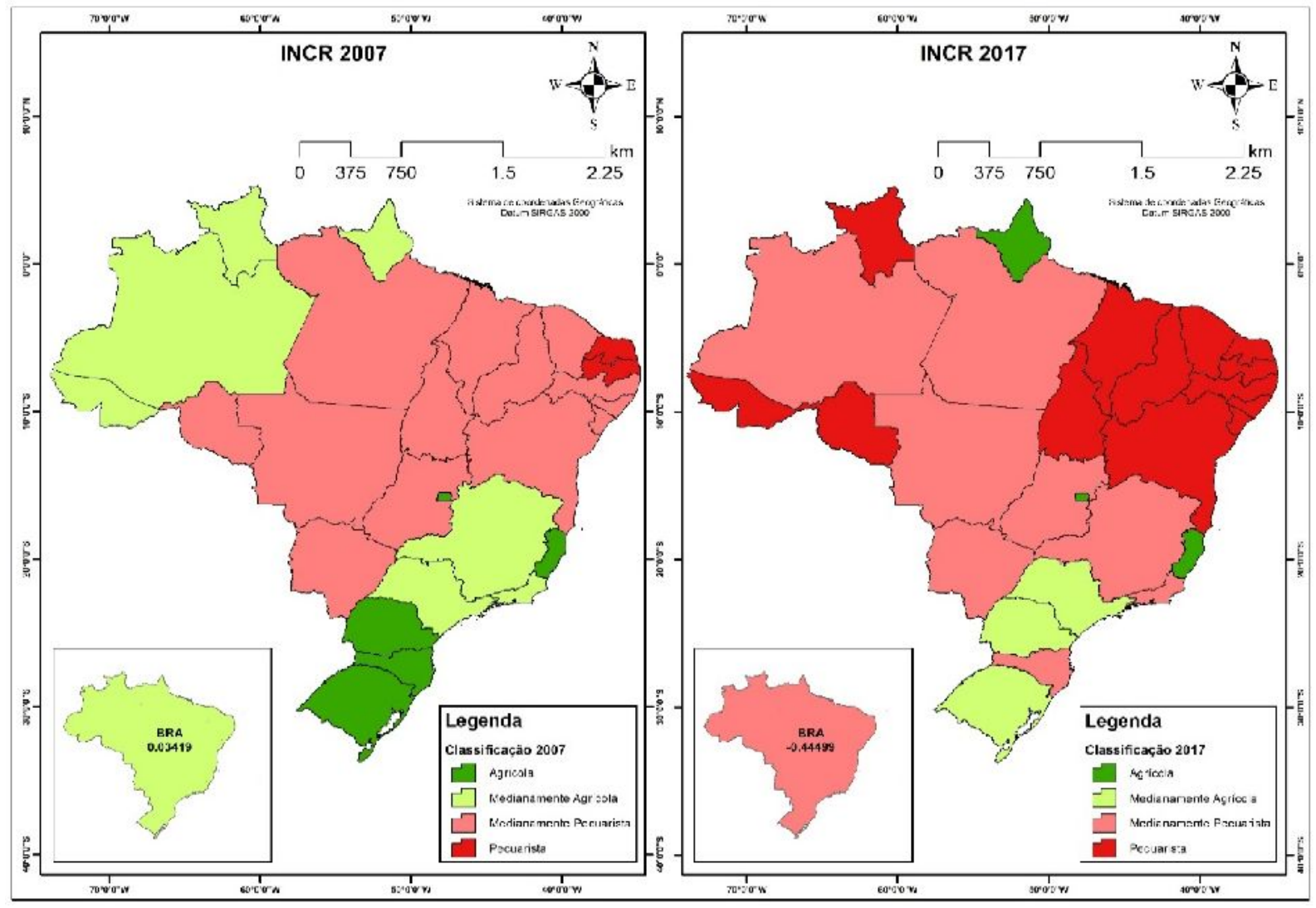

Fonte: dados da pesquisa. 
Os cinco estados que mais receberam financiamentos agrícolas foram: Paraná, Rio Grande do Sul, São Paulo, Minas Gerais e Mato Grosso, foram aplicados $\mathrm{R} \$ 464$ bilhões, o que correspondeu a $72,12 \%$ do total de crédito agrícola aplicado no período de 2007 a 2017. Spolador e Lima (2009), analisando a concentração das aplicações de crédito agrícola no período de 2000 a 2007, obtiveram resultados similares, pois os estados com maiores participações foram: São Paulo, Rio Grande do Sul, Paraná e Minas Gerais. A partir desta análise, os resultados indicaram que os estados com maiores participações na produção agrícola recebem uma parcela de crédito superior se comparados a outros estados que possuem uma representatividade inferior das suas lavouras.

No caso dos financiamentos destinados a pecuária os cinco estados que tiveram maior destaque, foram: Minas Gerais, Goiás, Paraná, São Paulo e Rio Grande do Sul, foram aplicados R $\$ 178$ bilhões, correspondendo a 54,41\% do total de crédito pecuário aplicado no período.

Apesar da região Norte não ter obtido destaque na absorção dos maiores valores de financiamento pecuário, avaliando o comportamento do rebanho bovino no período compreendido entre os anos de 1974 a 2010, a região Norte já apresentava grande potencial neste segmento. Em face disso, ao longo dos anos houve um aumento na disponibilidade de recursos para o financiamento em pecuária, sobretudo a partir da década de 1990. Por outro lado, as regiões detentoras dos maiores volumes de crédito (Regiões Sul e Sudeste) no referido período, estiveram pouco relacionadas com o crescimento do rebanho, indicando a necessidade de estudos voltados para identificação de outros fatores que podem estar envolvidos na relação entre a disponibilidade de crédito e a aquisição de animais (SILVA NETO et al., 2011).

Considerando o volume total de recursos (crédito agrícola + crédito pecuário), verificou-se que $72,41 \%$ de todo o valor aplicado no período foi alocado em apenas seis estados (PR, RS, SP, MG, GO e MT). Nota-se que as regiões que detém uma agricultura tecnologicamente superior, são as mesmas que absorvem maiores parcelas de crédito se comparadas com regiões menos desenvolvidas, havendo, portanto, a necessidade de maiores estímulos visando o aumento da produtividade onde a agropecuária é incipiente, através da adoção de níveis tecnológicos mais elevados e o uso de estratégias que promovam ações integradas e comprometidas com a elevação da modernização agrícola, minimizando ainda possíveis impactos negativos ao meio ambiente (REBELLO et al., 2008).

Em relação às estimativas do Índice de Gini para as operações e valor das contratações de crédito agrícola, pecuária e total, os resultados da Tabela 2 evidenciam que há uma elevada concentração nos recursos relacionados ao crédito agrícola, pois os valores médios deste segmento ficaram na faixa de 0,65 e 0,72 para o número de operações e valor, respectivamente. As médias que correspondem ao crédito pecuário ficaram na faixa de 0,53 e 0,60 para o número de operações e valor. Isso mostra que esta linha de financiamento é concentrada, porém em menor proporção comparativamente ao financiamento de atividades agrícolas. Quando se observa o índice calculado para o valor total de crédito empregado a cada ano, percebe-se que ao longo do tempo a concentração tem diminuído. O número total de operações por sua vez, não apresentou mudanças significativas com o passar dos anos, pois os valores oscilaram entre 0,50 e 0,60 no período em análise. 
TABELA 2. Evolução do Índice de Gini das operações de crédito agrícola, pecuário e total no Brasil, 2007-2017.

\begin{tabular}{c|cccc|cc}
\hline \multirow{2}{*}{ Ano } & \multicolumn{2}{c|}{ Crédito Agrícola } & \multicolumn{2}{c|}{ Crédito Pecuário } & \multicolumn{2}{c}{ Total } \\
\cline { 2 - 7 } & Operações & Valor & Operações & Valor & Operações & Valor \\
\hline $\mathbf{2 0 0 7}$ & 0,692986 & 0,74248 & 0,5459 & 0,611316 & 0,557776 & 0,700939 \\
$\mathbf{2 0 0 8}$ & 0,729742 & 0,746436 & 0,50624 & 0,644147 & 0,605784 & 0,711674 \\
$\mathbf{2 0 0 9}$ & 0,732839 & 0,746499 & 0,502175 & 0,656725 & 0,608574 & 0,716167 \\
$\mathbf{2 0 1 0}$ & 0,727584 & 0,739673 & 0,504262 & 0,645611 & 0,591936 & 0,702938 \\
$\mathbf{2 0 1 1}$ & 0,738079 & 0,741209 & 0,514105 & 0,643962 & 0,598686 & 0,702361 \\
$\mathbf{2 0 1 2}$ & 0,678498 & 0,731074 & 0,486702 & 0,630253 & 0,563323 & 0,689314 \\
$\mathbf{2 0 1 3}$ & 0,557084 & 0,680183 & 0,514654 & 0,586035 & 0,524292 & 0,620646 \\
$\mathbf{2 0 1 4}$ & 0,558252 & 0,696456 & 0,528197 & 0,554574 & 0,511911 & 0,612515 \\
$\mathbf{2 0 1 5}$ & 0,602286 & 0,709517 & 0,61025 & 0,561039 & 0,572333 & 0,615816 \\
$\mathbf{2 0 1 6}$ & 0,61232 & 0,710126 & 0,62476 & 0,553548 & 0,586279 & 0,612277 \\
$\mathbf{2 0 1 7}$ & 0,61398 & 0,699251 & 0,535724 & 0,584618 & 0,504414 & 0,626894 \\
\hline MÉDIA & $\mathbf{0 , 6 5 8 5 1 3 6 3 6}$ & $\mathbf{0 , 7 2 2 0 8}$ & $\mathbf{0 , 5 3 3 9 0 6 2 7 3}$ & $\mathbf{0 , 6 0 6 5 3}$ & $\mathbf{0 , 5 6 5 9 3 7 0 9 1}$ & $\mathbf{0 , 6 6 4 6 9}$ \\
\hline
\end{tabular}

Fonte: dados da pesquisa.

Sobre essa dinâmica da concentração de crédito para a agricultura, Spolador e Lima (2009) ao analisarem o Índice T de Theil (cujos objetivos são semelhantes ao do Índice de Gini) com base no valor da produção e área plantada, obtiveram resultados diferentes de acordo com a variável-base. Ao analisarem a distribuição do crédito fazendo relação com o valor da produção, os autores observaram que houve uma desconcentração no período compreendido entre 2000 a 2007 em todas as finalidades (custeio, investimento e comercialização). Por outro lado, ao utilizarem a área plantada como referência, notaram um crescimento da concentração, exceto para a finalidade de investimento, ao qual apresentou uma forte desconcentração independentemente da base utilizada.

Este fato, explicado pelos próprios autores, pode ser devido a mudanças no padrão distributivo de crédito, que antes era em grande parte destinado a regiões com pequenas propriedades muito produtivas e posteriormente passou a convergir para regiões que dispõem de menor produção em propriedades com áreas maiores.

A concentração do rebanho bovino é citada por Macedo (2006), cujos resultados mostram que as regiões Sul, Sudeste e Centro-Oeste detinham os maiores rebanhos se comparadas às demais regiões no ano de 2004. Apesar disso, nesse mesmo período, a região Norte obteve destaque na atividade pecuária por apresentar crescentes percentuais de participação do efetivo bovino em escala nacional. $O$ autor ressalta ainda, que o aumento da tomada de crédito pelos produtores rurais deve-se a aquisição de animais geneticamente superiores e investimentos em formação e manutenção de pastagens, por exemplo.

No caso da pecuária na região Norte, o estado mais representativo é o Pará, que de acordo com a base de dados do IBGE (2018), possui cerca de 20 milhões de cabeças de bovinos, ficando em quinto lugar no ranking nacional. Rondônia por sua vez, é o segundo estado com maior representatividade na região com um efetivo de 14 milhões, sendo classificado como o sexto maior rebanho bovino do País em 2017. Estudos como o de Santos et al. (2012) e Nascimento et al. (2011) intensificam as discussões sobre a dinâmica creditícia no estado do Pará e Amapá, cujas aplicações para o financiamento pecuário são mais acentuadas no primeiro, chegando a atingir mais que o dobro do volume de crédito concedido à agricultura. 
Diferentemente do observado no Amapá, onde a alocação de crédito não se concentra em um setor de produção específico, pois o fluxo das aplicações leva em consideração as demandas de mercado e os respectivos ciclos das atividades produtivas.

\section{CONCLUSÃO}

Os resultados indicam que há uma grande concentração na aplicação de recursos de crédito rural no Brasil, pois $71,42 \%$ de todo o volume aplicado no período de 2007 a 2017, se concentrou em apenas seis Unidades da Federação. O Índice de Gini confirma essa concentração para todos os setores, sobretudo em financiamentos agrícolas, que assumiu um valor médio de 0,72 em relação ao valor das operações. O Índice Normalizado de Crédito Rural (INCR) permitiu identificar que há padrões diferenciados de especialização quanto à aplicação do crédito, pois as regiões Norte e Nordeste tiveram os maiores financiamentos em pecuária. Situação oposta ao observado no Sudeste, onde os financiamentos são voltados principalmente para atividades agrícolas.

\section{REFERÊNCIAS}

ANTÃO, R. A. S.; CAMPANHOLO, T. O Crédito Rural no Contexto do Desenvolvimento Econômico e Social. Revista da Católica, v. 03, p. 01-14, 2011. Disponível em:<http://www.catolicaonline.br/article/download/228/1475>. Acesso em: 7 out. 2018.

ARAÚJO, P. F. C. Política de crédito rural: reflexões sobre a política Brasileira. Textos para discussão. Brasília - DF: CEPAL/ IPEA, 2011. Disponível em:<http://www.ipea.gov.br/portal/images/stories/PDFs/TDs/td_1555.pdf>. Acesso em: 7 out. 2018.

BANCO CENTRAL DO BRASIL. Anuário Estatístico do Crédito Rural. Disponível em: http://www.bacen.gov.br. Acesso em: 20 set. 2018.

BELICK, W. O financiamento da agropecuária brasileira no período recente. Instituto de Pesquisa Econômica Aplicada. 2015. (Texto para Discussão). Disponível em:< http://repositorio.ipea.gov.br/bitstream/11058/3407/1/td_2028.pdf>. Acesso em: 7 out. 2018.

FUNDAÇÃO GETÚLIO VARGAS. FGVDADOS: Informação Econômica On-line. Disponível em: < http://fgvdados.fgv.br > Acesso em: 30 ago. 2018.

HOFFMANN, R. Estatística para economistas. 2. ed. São Paulo: Livraria Pioneira Editora. 1991. 426p.

IBGE - Instituto Brasileiro de Geografia e Estatística. Sistema IBGE de Recuperação Automática, Produção Agrícola Municipal. Disponível em: <https://sidra.ibge.gov.br/pesquisa/ppm/quadros/brasil/2017> Acesso em: 20 de Out. 2018.

MACEDO, L. O. B. Modernização da Pecuária de Corte Bovina no Brasil e a Importância do Crédito Rural. Informações Econômicas. São Paulo. v. 36. n. 7. jul. 
2006. Disponível em:<ftp://ftp.sp.gov.br/ftpiea/publicacoes/seto2-0706.pdf>. Acesso em: 7 out. 2018.

NASCIMENTO, M. N. C. F.; SANTOS, M. A. S.; ALMEIDA, R. H. C. Evolução e distribuição espacial das aplicações de crédito rural no estado do Amapá na primeira década do século 21. PRACS: Revista Eletrônica de Humanidades do Curso de Ciências Sociais da UNIFAP, v. 4, p. 79-94, 2011. Disponível em:< https://periodicos.unifap.br/index.php/pracs/article/view/363>. Acesso em: 27 ago. 2018.

RAMOS, S. Y.; MARTHA JUNIOR, G. B. Evolução da política de crédito rural Brasileira. Embrapa Cerrados, Planaltina - DF, 2010. Disponível em:< https://www.embrapa.br/en/busca-de-publicacoes/-/publicacao/899862/evolucao-dapolitica-de-credito-rural-brasileira >. Acesso em: 27 ago. 2018.

REBELLO, F. K.; SANTOS, M. A. S.; SOUZA, D. M. F. Modernização da agricultura regional: contribuições do Banco da Amazônia no período de 1989 a 2007. Contexto Amazônico, Belém, v. 1, n. 10, p. 1-4, set. 2008. Disponível em:< http://www.bancoamazonia.com.br/images/arquivos/institucional/biblioteca/boletim/co ntexto_amazonico_10.pdf>. Acesso em: 27 ago. 2018.

SANTOS, M. A. S.; REBELLO, F. K.; A. C. SANTANA. A política de crédito rural no estado do Pará: Distribuição espacial e concentração das aplicações no período 2000-2010. Revista em Agronegócio e Meio Ambiente. v. 5, n. 3, p. 493-508. set./dez. 2012.

Disponível em:<http://periodicos.unicesumar.edu.br/index.php/rama/article/view/2115>. Acesso em: 27 ago. 2018.

SANTOS, W. B. O Papel das Políticas Governamentais na Modernização da Agricultura Brasileira. 2010.2 Disponível em:<https://pantheon.ufrj.br/bitstream/11422/2360/1/WBSantos.pdf>. Acesso em: 20 out. 2018.

SILVA NETO, W. A. N.; BACHA, C. J. C.; BACCHI, M. R. P. Evolução do financiamento rural para a pecuária e sua relação com a dinâmica regional dessa atividade no Brasil. Goiânia, GO: UFG-NEPEC-FACE, 2011. 24p. (Textos para Discussão, 24). Disponível em:<http://www.face.ufg.br/siteface_files/midias/original-td-024.pdf >. Acesso em: 20 out. 2018.

SOUZA, P. M.; NEY, M. G.; PONCIANO, N. J. Análise da distribuição dos financiamentos rurais entre os estabelecimentos agropecuários brasileiros. Revista de Economia e Sociologia Rural. Piracicaba. v. 53.n. 2. p. 251-270. abr.jun. 2015. Disponível em:<http://www.scielo.br/scielo.php?script=sci_arttext\&pid=S0103$20032015000200251>$. Acesso em: 20 out. 2018.

SOUZA, P. M.; BARBÉ, L. C. Desigualdades regionais na distribuição dos financiamentos do PRONAF: Uma análise do período de 1998 a 2012. Revista Econômica do Nordeste. Fortaleza. v. 45. p. 31-43. 2014. Disponível em:< https://ren.emnuvens.com.br/ren/article/view/497>. Acesso em: 20 out. 2018. 
SPOLADOR, H. F. S.; LIMA, R. A. S. Evolução da distribuição de crédito agrícola no Brasil entre unidades da federação no período de 2000 a 2007. In: Congresso Brasileiro da Sociedade de Economia , Administração e Sociologia Rural - SOBER, 47, 2009, Porto Alegre. Anais... Porto Alegre: SOBER, 2009, p. 1-19. Disponível em:<http://www.sober.org.br/anais/2009_45>. Acesso em: 20 out. 2018.

TOLEDO NETO, E. R., COUTO JUNIOR, A. F. Índice Normalizado de Agricultura e Pecuária (INAP) para caracterização da dinâmica de concessão de crédito rural em Goiás. In: Anais XV Simpósio Brasileiro de Sensoriamento Remoto SBSR, Curitiba, PR, Brasil, 30 de abril a 05 de maio de 2011, INPE p.176. Disponível

em:<http://marte.dpi.inpe.br/rep/dpi.inpe.br/marte/2011/07.12.17.29?mirror=urlib.net/ $w w w / 2011 / 03.29 .20 .55 \&$ metadatarepository=dpi.inpe.br/marte/2011/07.12.17.29.39 >. Acesso em: 20 out. 2018. 